MATEC Web of Conferences 1, 03008 (2012)

DOI: $10.1051 /$ matecconf $/ 20120103008$

(C) Owned by the authors, published by EDP Sciences, 2012

\title{
Camber Effects on the Power Harvesting from Piezoaeroelastic Systems
}

\author{
Abdessattar Abdelkefi ${ }^{1}$, Abdullah O Nuhait ${ }^{2}$, Ali H Nayfeh ${ }^{1}$, and Muhammad R Hajj ${ }^{1, a}$ \\ ${ }^{1}$ Department of Engineering Science and Mechanics, MC 0219, Virginia Polytechnic Institute and State University, Blacks- \\ burg, Virginia 24061, USA. \\ 2 Mechanical Engineering Department, King Saud University, Riyadh 11421, Saudi Arabia.
}

\begin{abstract}
We investigate the effects of the aerodynamic loads on the performance of piezoaeroelastic energy harvesters. The harvester consists of a rigid airfoil having a pitch and plunge degrees of freedom with a piezoelectric coupling attached to the plunge degree of freedom. The Unsteady Vortex Lattice Method is used to model the unsteady flow and predict the loads. An iterative scheme based on Humming's fourth order predictor-corrector method is employed to solve simultaneously and interactively the governing equations. The effects of varying the airfoil camber coefficient are determined. We demonstrate that increasing the camber does not necessarily increase the level of the harvested power.
\end{abstract}

\section{Introduction}

The use of aeroelastic vibrations to harvest energy has been the focus of many previous studies. De Marqui et al.[1] presented frequency domain piezoaeroelastic modeling and analysis of an unswept generator wing using the doubletlattice method to model the aerodynamic loads. Erturk et al.[2] determined theoretically and experimentally the effects of piezoelectric power generation on the linear flutter speed. Because power generation is most efficient from limit cycle oscillations, Abdelkefi et al.[3,4] noted that evaluating aeroelastic responses and power generation are best determined from nonlinear responses of the harvester. In fact, structures subjected to wind loads can undergo various responses [5], including bifurcations, limit cycle oscillations, internal resonances and chaotic motions.

The level of the harvested power is related to airfoil parameters and the freestream velocity. In this work, we focus on the effects of the aerodynamic loads on the performance of the energy harvester. The focus is on the effects of the camber of the airfoil on the harvested voltage, pitch and plunge motions.

\section{Mathematical Modeling}

The piezoaeroelastic system, considered in this work, consists of a rigid airfoil that is allowed to move with two degrees of freedom, as shown in Figure 1. The airfoil is supported by linear and nonlinear torsional and flexural springs with a piezoelectric coupling attached to the plunge degree of freedom. The governing equations of this system are written as $[2,4]$ :

$$
\begin{gathered}
m_{T} \ddot{h}+m_{W} x_{\alpha} b \ddot{\alpha}+c_{h} \dot{h}+k_{h}(h) h-\theta V=-\rho U^{2} b C_{n} \\
m_{W} x_{\alpha} b \ddot{h}+I_{\alpha} \ddot{\alpha}+c_{\alpha} \dot{\alpha}+k_{\alpha}(\alpha) \alpha=2 \rho U^{2} b^{2} C_{m} \\
C_{p} \dot{V}+\frac{V}{R}+\chi \dot{h}=0
\end{gathered}
$$

where $m_{T}$ is the total mass of the wing, including its sup-

\footnotetext{
a e-mail: mhajj@vt.edu
}

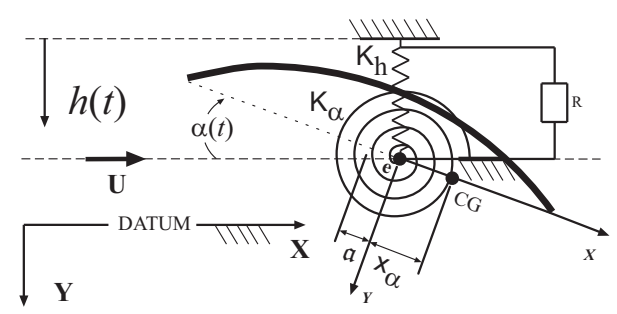

Fig. 1. Schematic of a cambered piezoaeroelastic system under uniform airflow.

port structure; $m_{W}$ is the wing mass alone; $I_{\alpha}$ is the mass moment of inertia about the elastic axis; $b$ is the half chord length; $x_{\alpha}$ is the dimensionless distance between the center of mass and the elastic axis; $c_{h}$ and $c_{\alpha}$ are, respectively, the plunge and pitch structural damping coefficients; $C_{n}$ and $C_{m}$ are, respectively, the normal force coefficient and the pitching moment coefficient about the elastic axis; $R$ is the load resistance; $U$ is the freestream velocity; $V$ is the voltage across this load resistance; $C_{p}$ is the capacitance of the piezoelectric layer; $\theta$ and $\chi$ are electromechanical coupling terms, and $k_{h}$ and $k_{\alpha}$ are the structural stiffness for the plunge and pitch motions, respectively. Here, we represent the stiffness for the plunge and pitch motions in polynomial forms respectively as

$$
\begin{aligned}
k_{\alpha}(\alpha) & =k_{\alpha 0}+k_{\alpha 2} \alpha^{2} \\
k_{h}(h) & =k_{h 0}+k_{h 2} h^{2}
\end{aligned}
$$

The three equations of motion are nondimensionalized using the following characteristic parameters: $l$ for length, $U$ for velocity, and $\frac{l}{U}$ for time. Using this nondimensional strategy, $h^{*}=\frac{h}{l}$ and $V^{*}=\frac{V}{V_{0}}$ where $V_{0}=\frac{\chi l}{C_{p}}$. Eliminating hats, the resulting nondimensional equations of motion are:

$$
\ddot{h}=\left[\frac{1}{r^{2}-m^{*} x_{\alpha}^{2}}\right]\left[-\frac{2 r^{2} c_{h}^{*}}{N_{e l} W} \dot{h}+\frac{4 x_{\alpha} m^{*} c_{\alpha}^{*}}{W} \dot{\alpha}-\frac{4 \sigma^{2} r^{2}}{N_{e l}^{2} W^{2}} h\right.
$$




$$
\begin{gathered}
+\frac{2 x_{\alpha} m^{*} r^{2}}{N_{e l} W^{2}} \alpha-\frac{4 \sigma_{2} \sigma^{2} r^{2}}{N_{e l}^{4} W^{2}} h^{3}+\frac{2 x_{\alpha} \sigma_{3} m^{*} r^{2}}{N_{e l} W^{2}} \alpha^{3} \\
\left.+\frac{4 \sigma_{5} r^{2}}{N_{e l}^{2} W^{2}} V-\frac{2 m^{*} r^{2}}{\pi \mu N_{e l}} C_{n}-\frac{4 m^{*} x_{\alpha}}{\pi \mu N_{e l}} C_{m}\right] \\
\ddot{\alpha}=\left[\frac{1}{r^{2}-m^{*} x_{\alpha}^{2}}\right]\left[\frac{4 x_{\alpha} c_{h}^{*}}{N_{e l}^{2} W} \dot{h}-\frac{8 c_{\alpha}^{*}}{N_{e l} W} \dot{\alpha}+\frac{8 \sigma^{2} x_{\alpha}}{N_{e l}^{3} W^{2}} h\right. \\
-\frac{4 r^{2}}{N_{e l}^{2} W^{2}} \alpha+\frac{8 \sigma_{2} \sigma^{2} x_{\alpha}}{N_{e l}^{5} W^{2}} h^{3}-\frac{4 \sigma_{3} r^{2}}{N_{e l}^{2} W^{2}} \alpha^{3} \\
\left.-\frac{8 x_{\alpha} \sigma_{5}}{N_{e l}^{3} W^{2}} V+\frac{4 m^{*} x_{\alpha}}{\pi \mu N_{e l}^{2}} C_{n}+\frac{8}{\pi \mu N_{e l}^{2}} C_{m}\right] \\
\dot{V}+\frac{2 \sigma_{6}}{N_{e l} W} V+\dot{h}=0
\end{gathered}
$$

where $m^{*}=\frac{m_{W}}{m_{T}}, c_{h}^{*}=\frac{c_{h}}{m_{T} w_{\alpha}}$ is the nondimensional plunge damping coefficient, $c_{\alpha^{*}}=\frac{c_{\alpha}}{m_{W} c^{2} w_{\alpha}}$ is the nondimensional pitch damping coefficient, $\mu=\frac{4 m_{W}}{\pi \rho c^{2}}$ is the mass ratio, $r^{2}=$ $\frac{4 I_{\alpha}}{m_{W} c^{2}}$ is the dimensionless square of the radius of gyration, $W=\frac{2 U}{c w_{\alpha}}$ is the reduced velocity, $\sigma=\frac{w_{h}}{w_{\alpha}}$ is the frequency ratio in which $w_{\alpha}=\sqrt{\frac{k_{\alpha 0}}{I_{\alpha}}}$ and $w_{h}=\sqrt{\frac{k_{h 0}}{m_{T}}}$ are the structural pitch and plunge natural frequencies, respectively. $N_{e l}$ is the number of elements in the aerodynamic model. $\sigma_{2}=$ $\frac{c^{2} k_{h 2}}{k_{h 0}}$ is the plunge spring coefficients ratio, $\sigma_{3}=\frac{k_{\alpha 2}}{k_{\alpha 0}}$ is the pitch spring coefficients ratio, $\sigma_{5}=\frac{\theta \chi}{m_{T} w_{\alpha}^{2} C_{p}}$ is the first nondimensional electromechanical coupling term, and $\sigma_{6}=$ $\frac{1}{R C_{p} w_{\alpha}}$ is the second nondimensional electromechanical coupling term. The characteristic length $l$ has been replaced by $c / N_{e l}$.

\section{Aerodynamic model}

The unsteady flow around a cambered plate is modeled using the unsteady vortex lattice method (UVLM). The flow is assumed to be incompressible and inviscid. The plate and its wake are represented by sheets of vorticity. The position of the sheet representing the plate is known and is called a bound vortex sheet. Each vortex is interpreted as an infinite-long vortex filament oriented in the normal direction of the plate. The position of the sheet representing the wake is not known in advance and is determined as part of the solution. This sheet deforms freely during the simulation, it assumes a force-free position. This sheet is called a free vortex sheet. These two vortex sheets are joined along the trailing edge of the plate. In this numerical method, these two vortex sheets are replaced by discrete vortices. Furthermore, the rigid plate is divided into $N_{e l}$ equal-length panels or piecewise straight line segments. In each panel, the vorticity distributed on each element is replaced by a single vortex of unknown circulation strength $\Gamma_{j}$ which is located at one quarter of the element length. The starting vortex is simulated by locating a vortex at the trailing edge of unknown circulation strength $\Gamma_{c}$. According to the Biot-Savart law, the magnitude of the induced velocity varies directly with the strength of the vortex and inversely with the distance between the vortex and point of interest. This law gives the velocity $\mathbf{W}$ at a point $\mathbf{r}$ due to an individual vortex point located at $\mathbf{r}_{\mathbf{0}}$ and which has a circulation $\Gamma(t)$ The total velocity field induced by these vortices satisfies the continuity equation, the no-penetration boundary condition on the plate, zero fluid velocity at infinity, unsteady Kutta condition at trailing edge of the rigid plate, the total circulation around the closed fluid line that encircles the plate and its wake is conserved (zero in this case), and the pressure is continuous in the wake. The no-penetraion boundary condition is imposed at one point of each panel which is named control point. This point is placed at the three-quarter point of the element. Furthermore, the nondimensional length of the element is equal to unity and hence the nondimensional chord of the plate is set equal to $N_{e l}$.

The unsteady Bernoulli's equation was used to compute the pressure jump $\Delta p$ across each element at its control point. For more details on the aerodynamic model, one is referred to the paper of Zedan and Nuhait[6].

\section{Camber implementation}

In this work, the effect of the camber is introduced using the following expression for a cambered four digit NACA airfoil to calculate the mean camber line If $x<L_{c}$

$$
y_{c}=\frac{c_{b}}{L_{c}}\left(2 L_{c} x-x^{2}\right)
$$

If not

$$
y_{c}=\frac{c_{b}}{\left(1-L_{c}\right)^{2}}\left(1-2 L_{c}+2 L_{c} x-x^{2}\right)
$$

where $c_{b}$ and $L_{c}$ represent respectively the maximum camber $\left(100 c_{b}\right.$ is the first of the four digits) and the location of the maximum camber $\left(10 L_{c}\right.$ is the second of the four digits)

\section{Predictor-corrector methodology}

To rewrite the equations of motion as a set of first order equations, we consider the following state variables

$$
X=\left[\begin{array}{l}
X_{1} \\
X_{2} \\
X_{3} \\
X_{4} \\
X_{5}
\end{array}\right]=\left[\begin{array}{l}
h \\
\dot{h} \\
\alpha \\
\dot{\alpha} \\
V
\end{array}\right]
$$

we rewrite the equations of motions as

$$
\dot{X}_{1}=X_{2}
$$

$$
\begin{array}{r}
\dot{X}_{2}=\left[\frac{1}{r^{2}-m^{*} x_{\alpha}^{2}}\right]\left[-\frac{2 r^{2} c_{h}^{*}}{N_{e l} W} X_{2}+\frac{4 x_{\alpha} m^{*} c_{\alpha}^{*}}{W} X_{4}-\frac{4 \sigma^{2} r^{2}}{N_{e l}^{2} W^{2}} h\right. \\
+\frac{2 x_{\alpha} m^{*} r^{2}}{N_{e l} W^{2}} X_{3}-\frac{4 \sigma_{2} \sigma^{2} r^{2}}{N_{e l}^{4} W^{2}} X_{1}^{3}+\frac{2 x_{\alpha} \sigma_{3} m^{*} r^{2}}{N_{e l} W^{2}} X_{3}^{3} \\
\left.+\frac{4 \sigma_{5} r^{2}}{N_{e l}^{2} W^{2}} X_{5}-\frac{2 m^{*} r^{2}}{\pi \mu N_{e l}} C_{n}-\frac{4 m^{*} x_{\alpha}}{\pi \mu N_{e l}} C_{m}\right]
\end{array}
$$


Table 1. Parameters of the piezoaeroelastic system

\begin{tabular}{cc}
\hline \hline$a$ & -0.6847 \\
$\sigma$ & 1.37 \\
$\mu$ & 29.21 \\
$x_{\alpha}$ & 0.33 \\
$m^{*}$ & 1 \\
$r^{2}$ & 1.49 \\
$c_{h}^{*}$ & 0.2 \\
$c_{\alpha}^{*}$ & 0.02 \\
$\sigma_{2}$ & 0 \\
$\sigma_{5}$ & 0.013 \\
$\sigma_{6}$ & 0.75 \\
\hline \hline
\end{tabular}

$$
\begin{gathered}
\dot{X}_{3}=X_{4} \\
\dot{X}_{4}=\left[\frac{1}{r^{2}-m^{*} x_{\alpha}^{2}}\right]\left[\frac{4 x_{\alpha} c_{h}^{*}}{N_{e l}^{2} W} X_{2}-\frac{8 c_{\alpha}^{*}}{N_{e l} W} X_{4}+\frac{8 \sigma^{2} x_{\alpha}}{N_{e l}^{3} W^{2}} X_{1}\right. \\
-\frac{4 r^{2}}{N_{e l}^{2} W^{2}} X_{2}+\frac{8 \sigma_{2} \sigma^{2} x_{\alpha}}{N_{e l}^{5} W^{2}} X_{1}{ }^{3}-\frac{4 \sigma_{3} r^{2}}{N_{e l}^{2} W^{2}} X_{3}{ }^{3} \\
\left.-\frac{8 x_{\alpha} \sigma_{5}}{N_{e l}^{3} W^{2}} X_{5}+\frac{4 m^{*} x_{\alpha}}{\pi \mu N_{e l}^{2}} C_{n}+\frac{8}{\pi \mu N_{e l}^{2}} C_{m}\right] \\
\dot{X}_{5}+\frac{2 \sigma_{6}}{N_{e l} W} X_{5}+X_{2}=0
\end{gathered}
$$

These equations of motion are integrated numerically to determine the harvested voltage, pitch and plunge responses To integrate these governing equations numerically, simultaneously, and interactively in the time domain, it is complicated to determine it directly because the aerodynamic loads and the motion of the plate are related at each time step. In fact, to predict the aerodynamic loads, we have to know the motion of the rigid plate, and to predict the motion of this plate, we need to know the aerodynamic loads. Consequently, an iterative scheme based on Humming's fourth order predictor-corrector method is employed [7].

\section{Effects of the camber on the flutter speed and the performance of the harvester}

Before investigating the impact of the aerodynamic loads when varying the camber on the harvested voltage, pitch and plunge amplitudes, we study their effects on the flutter speed. Using the parameters given in Table 1, the plotted curves in Figures 2 and 3 show the time histories of the harvested voltage when the reduced wind speed are set equal, respectively, to 3.88 (directly before flutter) and 3.9 (directly after flutter) for different considered cases of the camber when the camber value is set equal to 0.04 . In this work, the location of maximum camber is set to 0.6.

We note that when the reduced airspeed is equal to 3.88 the response of the harvested voltage decreases with time. Consequently, the system response is damped. This result occurs because the sum of the structure and aerodynamic damping are negative (resulting in a decay) and then the system response is stable. Increasing the wind speed to 3.9, a limit cycle oscillations is developed and then the positive damping leads to the appearance of unstable solutions.

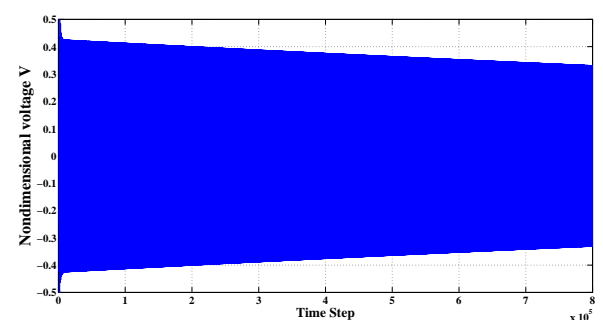

Fig. 2. Time histories of the nondimensional harvested voltage when $W=3.88$ and $c_{b}=0.04$.

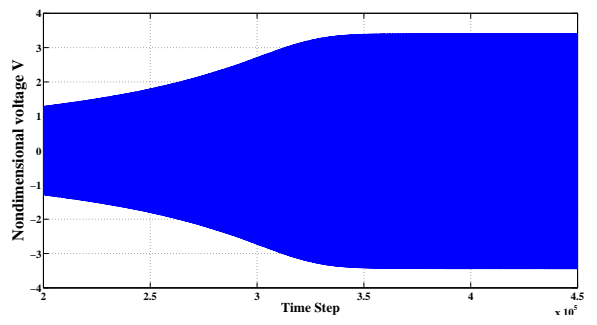

Fig. 3. Time histories of the nondimensional harvested voltage when $W=3.9$ and $c_{b}=0.04$.

We conclude that the flutter speeds for different values of the camber are between 3.88 and 3.9. The plotted curves in Figures 4, 5, and 6 show the variations of the nondimensional harvested voltage, nondimensional plunge, and pitch amplitudes when varying the camber when $\sigma_{3}=80$ and for three different values of the reduced velocity. We note that the increase of the $\left|c_{b}\right|$ is accompanied with a decrease on the system's outputs. Consequently, the configuration in which the camber is considered zero (flat plate), the harvested power is more important. For example, when $W=3.9$, the nondimensional harvested voltage decrease by $14 \%$ ( $31 \%$ in the harvested power) when the camber change from zero to 0.05 .

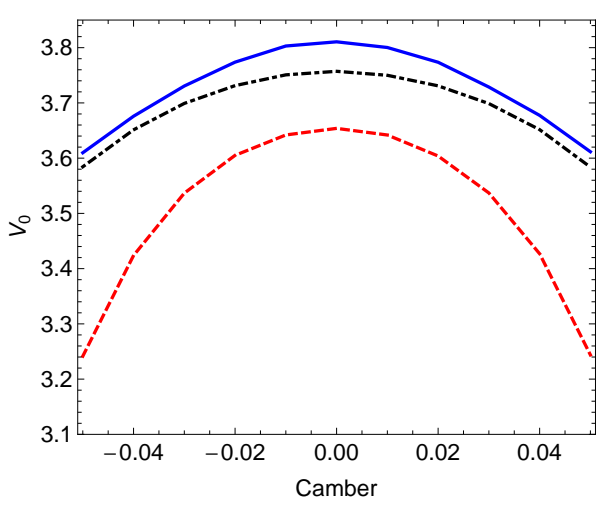

Fig. 4. Variations of the nondimensional harvested voltage with the camber when $\sigma_{3}=80$ and $W=3.9$ (red dashed line), $W=4$ (black dot-dashed line), and $W=4.2$ (blue solid line).

\section{Conclusions}

In this work, we have studied the effects of the aerodynamic loads on the performance of piezoaeroelastic systems. This harvester consists of a rigid airfoil supported 


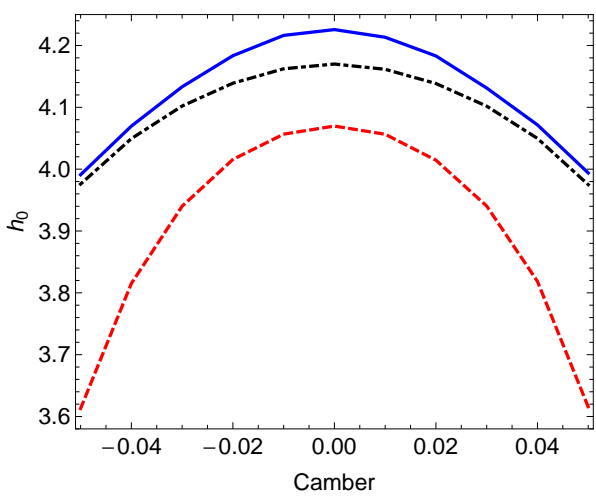

Fig. 5. Variations of the nondimensional plunge amplitude with the camber when $\sigma_{3}=80$ and $W=3.9$ (red dashed line), $W=4$ (black dot-dashed line), and $W=4.2$ (blue solid line).

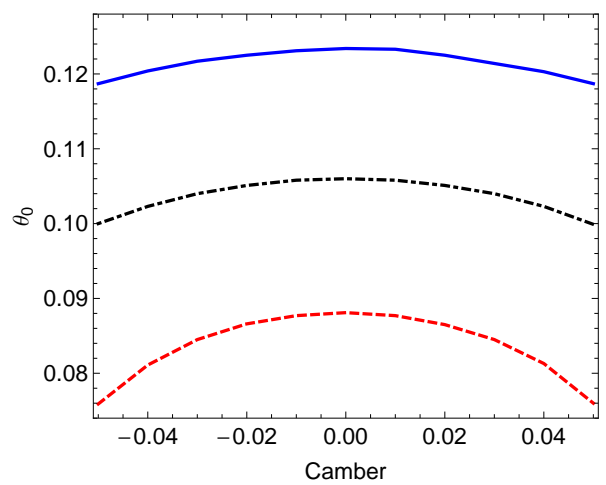

Fig. 6. Variations of the pitch amplitude with the camber when $\sigma_{3}=80$ and $W=3.9$ (red dashed line), $W=4$ (black dot-dashed line), and $W=4.2$ (blue solid line).

by flexural and torsional springs with a piezoelectric coupling attached to the plunge degree of freedom. The aerodynamic loads effects are investigated through varying the camber of the rigid airfoil. We have used a two dimensional Unsteady Vortex Lattice Method to model the unsteady flow. Because the aerodynamic loads and the motion of the plate are related at each time step, we used an iterative scheme based on Humming's fourth order predictorcorrector method to solve simultaneously and interactively the governing equations. The results show that varying the camber has a negligible influence on the flutter speed. On the other hand, the increase of the camber results in a decrease on the harvested power which can attain $31 \%$.

\section{References}

1. De Marqui C., Vieira, W. G. R., Erturk, A. and Inman, D. J., "Modeling and analysis of piezoelectric energy harvesting from aeroelastic vibrations using the doublet-lattice method," Journal of Vibration and Acoustics 133011003 (2011).

2. Erturk, A., Vieira, W. G. R., De Marqui, C. and Inman, D. J., "On the energy harvesting potential of piezoaeroelastic systems," Applied Physics Letters 96, 184103 (2010).

3. Abdelkefi, A., Nayfeh, A. H. and Hajj, M. R., "Modeling and analysis of piezoaeroelastic energy har- vesters," Nonlinear Dynamics, DOI 10.1007/s11071011-0035-1 (2011).

4. Abdelkefi, A., Nayfeh, A. H. and Hajj, M. R., "Design of piezoaeroelastic energy harvesters ," Nonlinear Dynamics, DOI 10.1007/s11071-011-0233-x (2011).

5. Dowell, E. H. and Tang, D., "Nonlinear aeroelasticity and unsteady aerodynamics," AIAA Journal 40, 16971707 (2002).

6. Nuhait, A. O. and Zedan, M. F., "Numerical simulations of unsteady flow induced by a flat plate moving near ground," Journal of Aircraft 30, 611617. doi:10.2514/3.46389 (1993).

7. Carnahan, B., Luther, H. A. and Wilkes, J. O. 1969. "Applied numerical methods," Wiley, New York (1969). 